ISSN 0103-9954

\title{
INFLUÊNCIAS DA ESPÉCIE, TRATAMENTO PRESERVATIVO E ADESIVOS NAS PROPRIEDADES FÍSICAS DO BAMBU LAMINADO COLADO
}

\author{
INFLUENCES OF SPECIES, PRESERVATIVE TREATMENT AND ADHESIVES ON PHYSICAL \\ PROPERTIES OF LAMINATED BAMBOO LUMBER
}

\author{
Rafael Amorim Rosa ${ }^{1}$ Juarez Benigno Paes ${ }^{2}$ Pedro Gutemberg de Alcântara Segundinho ${ }^{3}$ \\ Graziela Baptista Vidaurre ${ }^{4}$ Ana Karla Freire de Oliveira ${ }^{5}$
}

\begin{abstract}
RESUMO
Este trabalho teve como objetivo avaliar as propriedades físicas de laminados colados de bambu quanto à espécie, tratamento preservativo e adesivos utilizados. Foram produzidas ripas com os bambus das espécies gigante (Dendrocalamus giganteus) e vulgar (Bambusa vulgaris) com idade superior a três anos. Uma parte das ripas foi imersa em água e a outra em solução de octaborato de dissódio tetra-hidratado $\left(\mathrm{Na}_{2} \mathrm{~B}_{8} \mathrm{O}_{13} \cdot 4 \mathrm{H}_{2} \mathrm{O}\right)$ - Timbor, com $2 \%$ de concentração, durante 15 dias. Depois de tratadas e secas ao ar, as ripas foram transformadas em taliscas com dimensões de $0,5 \times 3,0 \times 47 \mathrm{~cm}$ (espessura $\times$ largura $\mathrm{x}$ comprimento) e empregadas na confecção dos bambus laminados colados (BLCs). Os adesivos utilizados na colagem das taliscas foram à base de emulsão de isocianato polimérico (EPI), melamina-ureia-formaldeído (MUF), acetato de polivinila lincado (PVAc) e resorcinol-formaldeído (RF). Os tratamentos preservativos e os adesivos não influenciaram as densidades dos BLCs confeccionados com bambu-gigante. Para o bambu-vulgar, as densidades dos BLCs foram afetadas pelo tipo de adesivo. Observou-se que os BLCs confeccionados com bambu-gigante tiveram maior estabilidade dimensional que aqueles produzidos com bambu-vulgar. Os adesivos MUF e RF conferiram maior estabilidade dimensional aos BLCs produzidos com ambas as espécies de bambu. Os BLCs aderidos com EPI ou PVA tiveram maior delaminação, independentemente da espécie de bambu e tratamento preservativo empregado, não sendo indicados para usos externos.
\end{abstract}

Palavras-chave: Dendrocalamus giganteus; Bambusa vulgaris; adesivos; caracterização física.

\section{ABSTRACT}

This work aimed to evaluate the physical properties of laminated bamboo lumber - LBL in relation to species, preservative treatments and adhesive employed. Laths of Dendrocalamus giganteus and Bambusa vulgaris bamboos older than three years were produced cross-linked polyvinil acetate (PVAc) the other in solution of disodium octaborate tetrahydrate $\left(\mathrm{Na}_{2} \mathrm{~B}_{8} \mathrm{O}_{13} 4 \mathrm{H}_{2} \mathrm{O}\right)$ - Timbor, with $2 \%$ concentration, during 15 days. After treated and air season, the laths were transformed into strips with dimensions of $0.5 \mathrm{x}$

1 Engenheiro Industrial Madeireiro, Msc., Programa de Pós-Graduação em Ciências Florestais, Universidade Federal do Espírito Santo, CEP 29550-000, Jerônimo Monteiro (ES), Brasil. rafaelrosa.001@gmail.com

2 Engenheiro Florestal, Dr., Professor do Departamento de Ciências Florestais e da Madeira, Universidade Federal do Espírito Santo, Av. Governador Lindemberg, 316, Centro,CEP 29550-000, Jerônimo Monteiro (ES), Brasil. jbp2@uol.com.br

3 Engenheiro Civil, Dr., Professor do Departamento de Ciências Florestais e da Madeira, Universidade Federal do Espírito Santo, Av. Governador Lindemberg, 316, Centro,CEP 29550-000, Jerônimo Monteiro (ES), Brasil. p_gutemberg2001@yahoo.com.br

4 Engenheiro Florestal, Dr., Professora do Departamento de Ciências Florestais e da Madeira, Universidade Federal do Espírito Santo, Av. Governador Lindemberg, 316, Centro, CEP 29550-000, Jerônimo Monteiro (ES), Brasil. grazividaurre@gmail.com

5 Designer, Dr., Professora do Departamento de Desenho Industrial, Centro de Letras e Artes, Universidade Federal do Rio de Janeiro, Av. Pedro Calmon, 550, $6^{\circ}$ andar,Cidade Universitária, CEP 21941901, Rio de Janeiro (RJ), Brasil.anadesigner2000@yahoo.com.br

Recebido para publicação em 12/08/2013 e aceito em 10/11/2014

Ci. Fl., v. 26, n. 3, jul.-set., 2016 
$3.0 \times 45 \mathrm{~cm}$ (thickness $\mathrm{x}$ width $\mathrm{x}$ length) and employed in the manufacture of laminated bamboo lumbers. The adhesives used for gluing the strips were the basis of polymer isocyanate emulsion (PIE), melamine urea formaldehyde (MUF), polyvinyl acetate cross (PVAc) and resorcinol formaldehyde (RF). The preservative treatments and the adhesives did not influence the densities of LBLs manufactured with Dendrocalamus giganteus. For the Bambusa vulgaris, the densities of LBLs were affected by the type of adhesive. It was observed that the LBLs manufactured with Dendrocalamus giganteus had a higher dimensional stability than those produced with the Bambusa vulgaris. The MUF and RF adhesives have given greater dimensional stability to the BLCs produced with both species of bamboo. The LBLs adhered with EPI or PVA had greater delamination, regardless the species of bamboo and the preservative treatment, not being suitable for external uses.

Keywords: Dendrocalamus giganteus; Bambusa vulgaris; adhesives; physical characterization.

\section{INTRODUÇÃO}

Relatos arqueológicos já indicaram a utilização do bambu há cinco mil anos. Nas ruínas de Hemodu e Shishan, província de Zhejiang na China, foram descobertas esteiras e cestas produzidas com bambu. Já a sua utilização naquele país começou a ser relatada somente entre 1600 e 1100 anos A.C. (VAZ, 2010).

Estima-se que um bilhão de pessoas dependem diretamente ou indiretamente do bambu para o seu sustento. No ano de 2004, o consumo de derivados dessa planta chegou a 10 bilhões de dólares em todo o mundo, podendo duplicar até 2015. Em países latino-americanos e orientais, o bambu é utilizado na construção civil, artesanato, indústria moveleira, de celulose, alimentícia e nos setores químicos e de energia (GARCIA, 2010).

O bambu possui características desejáveis de resistência, flexibilidade, facilidade de produção, maturação e crescimento rápidos, o que proporciona a sua utilização para diversos fins, tais como, produção de móveis e utilização estrutural (PAES et al., 2010). Obataya et al. (2007) apontam que, além da flexibilidade do bambu, suas propriedades de dureza e rigidez podem ser comparadas com as de madeiras de folhosas.

No entanto, para o emprego industrial do bambu, em decorrência da presença de amido em suas células parenquimáticas, o que proporciona o ataque da broca do bambu (Dinoderus minutus), há a necessidade da lixiviação do amido pela água (MORÁN UBIDIA, 2002), da modificação térmica do amido (COLLA, 2010; BRITO, 2013) ou da aplicação de substâncias químicas (SANTOS; LOPES, 1998; AZZINI; BERALDO, 2001; TIBURTINO, 2012) para prevenir sua deterioração. Segundo Morán Ubidia (2002), a imersão em água é um dos métodos de tratamento preservativos mais utilizados em países que utilizam o bambu como matéria-prima industrial. Para Pereira e Beraldo (2008), a duração do tratamento por imersão em água pode variar de quatro a sete semanas.

No Brasil, entretanto, as informações pertinentes à utilização e industrialização do bambu ainda são incipientes, tornando-o uma matériaprima destinada ao artesanato tradicional e às estruturas temporárias (GARCIA, 2010). Desta forma, para promover o emprego industrial do bambu, é necessário o desenvolvimento de novos produtos e a realização de diferentes testes de resistência mecânica e biológica. Com relação a sua durabilidade natural, são indispensáveis pesquisas para obter os tratamentos preservativos mais adequados para este material, de modo a garantir estruturas e elementos mais duráveis.

Um mercado que está se expandindo mundialmente é o de laminados de bambu, que registrou aumento do consumo nos últimos anos. Contudo, é necessário o avanço em pesquisas, aumento de financiamentos, inovação tecnológica e políticas públicas para suprir esta demanda (GARCIA, 2010).

Dentre os painéis produzidos com bambu, aquele conhecido internacionalmente como Laminated Bamboo Lumber - LBL ou Bambu Laminado Colado - BLC pode ser definido como um painel formado por lâminas de bambu serradas, conhecidas como taliscas, e coladas. A produção do BLC teve início em função da demanda de materiais destinados à construção civil, consequência da abertura econômica da China por volta de 1980 (RIVERO, 2003).

Em uma ripa de bambu, ou seja, em uma peça que ainda não foi usinada, as faces externas e internas são compactas, lisas e cobertas com 
uma camada de cera com baixa afinidade à água e aos adesivos. As propriedades da parte central das taliscas são diferentes daquelas da parte interna e externa da ripa. A parte central é a principal na utilização industrial do bambu. A diferença na estrutura dessas três partes provoca diferenças evidentes na densidade, teor de umidade, retratibilidade, resistência mecânica e adesão. Tais características levam a influências negativas sobre o processamento e a utilização das taliscas, como por exemplo, as faces internas e externas possuem baixa aderência com adesivos, enquanto a parte central demonstra alta capacidade de ligação (QISHENG et al., 2002). Por isto, para a confecção do BLC, as camadas internas e externas devem ser removidas, de acordo com a metodologia empregada por Rivero (2003), Oliveira (2005) e Brito (2013).

Normalmente, as espécies de bambu mais utilizadas para a produção do BLC são: bambugigante (Dendrocalamus giganteus), bambu-vulgar ou comum (Bambusa vulgaris), bambu-guadua (Guadua angustifolia), bambu-tali (Gigantochloa apus), bambu-mossô (Phyllostachys pubescens) e bambu-chinês (Dendrocalamus latiflorus), que podem ser plantadas e exploradas comercialmente em várias regiões do Brasil (MOIZÉS, 2007).

Em função das premissas levantadas e considerando a importância do bambu laminado colado - BLC para os mais variados fins, este trabalho teve como objetivo avaliar as propriedades físicas de laminados colados de bambu em relação à espécie, tratamento preservativo e adesivos utilizados.

\section{MATERIAL E MÉTODOS}

\section{Espécies, procedência e coleta dos bambus}

Foram utilizadas duas espécies de bambus, vulgar (Bambusa vulgaris Schrad. Ex J.C.Wendl) e gigante (Dendrocalamus giganteus Wall. Ex Munro), com idade superior a três anos, coletados em touceiras que cresciam no município de Jerônimo Monteiro, sul do estado do Espírito Santo. O Bambusa vulgaris foi obtido na latitude $20^{\circ} 48^{\prime} 27,75^{\prime \prime} \mathrm{S}$ e longitude $41^{\circ} 24^{\prime} 38,76^{\prime \prime} \mathrm{O}$, enquanto o Dendrocalamus giganteus foi colhido na latitude $20^{\circ} 46^{\prime} 8,69^{\prime \prime} \mathrm{S}$ e longitude $41^{\circ} 22^{\prime} 14,27^{\prime \prime} \mathrm{O}$.

O corte do bambu foi realizado a $20 \mathrm{~cm}$ do solo, logo acima do primeiro nó, como recomendado por Santos e Lopes (1998), para não permitir a entrada de água das chuvas, o que pode causar a morte dos rizomas e assim, não emitir mais brotos. Foram retirados de sete a oito indivíduos para cada espécie. Os colmos de bambu-vulgar possuíam, em média, $36,8 \mathrm{~cm}$ de diâmetro a altura do peito (DAP), $0,9 \mathrm{~cm}$ de espessura de parede e altura total de 20 $\mathrm{m}$. Os colmos de bambu-gigante tinham, em média, 39,6 $\mathrm{cm}$ de DAP, $1,3 \mathrm{~cm}$ de espessura de parede $\mathrm{e}$ altura total de $39,6 \mathrm{~m}$.

Os colmos foram serrados com comprimento de $1,40 \mathrm{~m}$, tendo cada um deles originado 12 a 16 seções. As secções foram selecionadas em função da espessura da parede dos colmos (mínimo de 0,70 $\mathrm{cm}$ ) e seccionadas em comprimentos de $0,70 \mathrm{~m} \mathrm{e}$ divididas longitudinalmente em quatro ripas. Cada duas delas foram destinadas a um dos tratamentos preservativos propostos.

Empregaram-se 60 amostras para cada um dos seguintes ensaios: densidade, curva de secagem e retratibilidade, conforme metodologia adaptada da Norma Brasileira Regulamentadora - NBR 7190 da Associação Brasileira de Normas Técnicas - ABNT (1997).

\section{Tratamento preservativo dos colmos}

Uma parte das ripas recebeu tratamento preservativo pela imersão prolongada em água e a outra em uma solução de octaborato de dissódio tetra-hidratado $\left(\mathrm{Na}_{2} \mathrm{~B}_{8} \mathrm{O}_{13} \cdot 4 \mathrm{H}_{2} \mathrm{O}\right)$, com $2 \%$ de concentração. A solução é conhecida popularmente como Timbor, sendo constituída pela reação do ácido bórico $\left(\mathrm{H}_{3} \mathrm{BO}_{3}\right)$ com o borato de sódio $\left(\mathrm{Na}_{2} \mathrm{~B}_{4} \mathrm{O}_{7} \cdot 10 \mathrm{H}_{2} \mathrm{O}\right.$ - bórax) na proporção de $1: 1,54$, respectivamente (LEPAGE et al., 1986). Ambos os tratamentos tiveram duração de 15 dias.

Para a imersão, tanto em água quanto em Timbor, foram confeccionados três tanques com placas de compensado de $14 \mathrm{~mm}$ de espessura e, posteriormente, revestidos com lona plástica. Os tanques possuíam dimensões de 53,7 x 40 × 200 $\mathrm{cm}$ (altura $\mathrm{x}$ largura $\mathrm{x}$ comprimento). Dois tanques foram reservados para a imersão em água e um para imersão em Timbor. Nos tanques com água foi necessário fazer a troca da mesma a cada dois dias por causa da fermentação do amido retirado pela água. Já o tanque com Timbor foi preenchido com 340 litros de água. Foram empregados $5,24 \mathrm{~kg}$ de bórax e 3,40 kg de ácido bórico a fim de atender à proporção proposta por Lepage et al. (1986). 


\section{Confecção e secagem das taliscas}

Para a usinagem das taliscas foi seguida a metodologia empregada por Rivero (2003), Oliveira (2005) e Brito (2013). Primeiramente, foi ajustada a largura das taliscas em uma serra circular e, em seguida, as taliscas brutas foram passadas em uma plaina desempenadeira para a retirada da face interna. Por fim, utilizou-se novamente a serra circular para a retirada da face externa e obtiveram-se taliscas com dimensões de $0,5 \times 3,0$ x $47 \mathrm{~cm}$ (espessura x largura x comprimento). A retirada das camadas internas e externas é justificada em função da propriedade impermeabilizante, o que poderia prejudicar a penetração dos adesivos. As camadas internas e externas das taliscas foram identificadas para que, na montagem do bambu laminado colado (BLC), não ficasse justaposta uma camada externa com externa ou uma camada interna com interna do bambu, pois isto poderia provocar ruptura em região de menor resistência (camada interna com interna).

Após serem produzidas, as taliscas foram secas em estufa durante cinco dias. No primeiro dia, utilizou-se a temperatura de $40^{\circ} \mathrm{C}$, no segundo de $60^{\circ} \mathrm{C}$, terceiro de $80^{\circ} \mathrm{C}$ e no quarto e quinto dia de 103 $\pm 2^{\circ} \mathrm{C}$. Depois de secas, as taliscas permaneceram durante 72 horas em um ambiente climatizado (20 \pm $2{ }^{\circ} \mathrm{C}$ de temperatura e $65 \pm 5 \%$ de umidade relativa) para estabilizar a umidade em $12 \%$.

\section{Adesivos utilizados na produção dos BLCs}

As taliscas foram aderidas com adesivos à base de emulsão de isocianato polimérico (EPI), melamina-ureia-formaldeído (MUF), acetato de polivinila lincado (PVAc) ou resorcinol-formaldeído (RF), os quais são normalmente empregados para colagem de laminados de madeira, e prensadas a frio em uma prensa hidráulica, dando origem aos BLCs (Figura 1). Após a prensagem, os BLCs foram climatizados por 24 horas, sob as mesmas condições citadas e confeccionados os corpos de prova necessários à caracterização física do laminado colado.

$\mathrm{O}$ adesivo RF empregado foi o CascophenRS126-M produzido pela empresa Momentive Speciality Chimicals Inc., que é bicomponente, utilizado juntamente com o endurecedor FM-60-M. $\mathrm{Na}$ colagem das lâminas, foi utilizada a gramatura média de 400 g.m.-2 , pressão de 1,0 MPa e tempo de prensagem de sete horas.

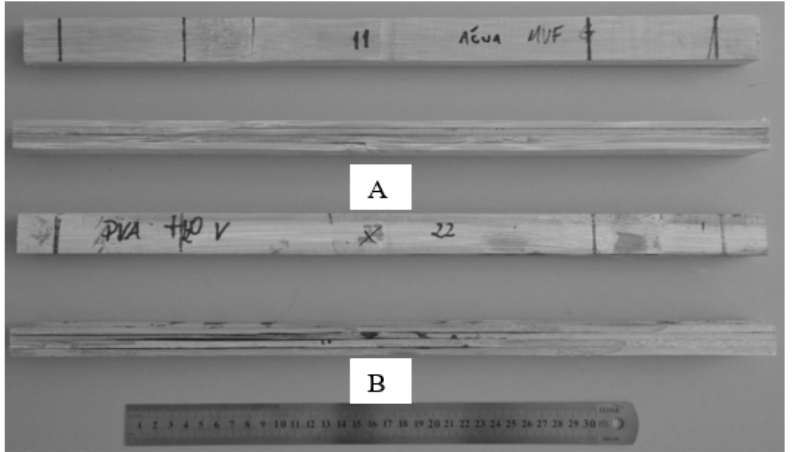

FIGURA 1: Bambu laminado colado - BLC produzido com bambu-gigante (A) e bambu-vulgar (B).

FIGURE 1: Laminated bamboo lumber - LBL of Dendrocalamus giganteus (A) and Bambusa vulgaris (B).

O PVAc utilizado foi o 9595 produzido pela empresa AkzoNobel, trata-se de um adesivo monocomponente à base de acetato de polivinila lincado. Na colagem das taliscas, foi utilizada a gramatura de 150 g.m ${ }^{-2}$, pressão de 1,0 MPa e tempo de prensagem de três horas.

O EPI utilizado foi o 1911 juntamente com o catalizador 1999, ambos produzidos pela empresa AkzoNobel. Na colagem, utilizou-se a gramatura média de 225 g.m-2 , pressão de 1,0 MPa e tempo de prensagem de uma hora.

A resina MUF empregada foi a 1242 misturada ao endurecedor 2542, ambos também fabricados pela empresa AkzoNobel. Na colagem foi utilizada a gramatura média de $425 \mathrm{~g} . \mathrm{m}^{-2}$, pressão de 1,0 MPa e tempo de prensagem de três horas.

\section{Ensaios de densidade básica, estabilidade dimensional e delaminação dos BLCs}

Para a realização dos ensaios de densidade básica e de estabilidade dimensional dos BLCs, seguiram-se as recomendações da American Society for Testing and Materials - ASTMD -143 (2005). Apesar de não existir direção radial no bambu por causa da ausência de raios em sua estrutura anatômica, adotaram-se os três planos de estudo da madeira para o bambu, ao considerar a largura da amostra como direção tangencial e a espessura como radial, esperando-se alcançar o melhor entendimento dos resultados.

O ensaio de delaminação foi realizado conforme as recomendações da American Institute of Timber Construction - AITC T110 (2007), que 
dispõem da avaliação de adesivos para uso estrutural de produtos de madeira destinados ao uso externo. A norma determina que a delaminação não deva exceder a $5 \%$ (madeira de coníferas) e $8 \%$ (madeira de folhosa) do comprimento da linha de cola.

A avaliação da delaminação consistiu em dispor as amostras em uma autoclave em três ciclos de vácuo e pressão de 8 horas cada. Em cada ciclo, aplicou-se um vácuo de $75 \pm 10 \mathrm{kPa}$ durante 2 horas, seguido de pressão de $540 \pm 20 \mathrm{kPa}$ durante 2 horas e, após este tempo, foi repetido o procedimento, totalizando 4 horas de vácuo e 4 horas de pressão por ciclo. Ao término de cada ciclo de 8 horas, as amostras foram secas ao ar livre durante 88 horas antes de iniciar um novo ciclo de vácuo e pressão e, também ao término do terceiro ciclo. Ao final do ensaio de delaminação, previu-se o comportamento da combinação do BLC exposto ao exterior, por meio da porcentagem de delaminação $(\% \mathrm{D})$, que é obtida nas duas faces de topo dos corpos de prova, de acordo com a Equação 1.

$$
\% \mathrm{D}=\frac{\left(\frac{\mathrm{d}_{1}+\mathrm{d}_{2}}{2}\right)}{d_{\text {total }}} \times 100
$$

Em que: $\% \mathrm{D}=$ Porcentagem da delaminação; $\mathrm{d}_{\mathrm{n}}=$ Delaminação na face $\mathrm{n}$ do corpo de prova $(\mathrm{cm}) ; \mathrm{d}_{\text {total }}$ $=$ Dimensão do corpo de prova $(\mathrm{cm})$.

\section{Análise estatística e avaliação dos resultados}

$\mathrm{Na}$ avaliação da densidade básica e da retratibilidade das espécies de bambu foi utilizada estatística descritiva, em que os valores foram avaliados em função da média aritmética e por meio de gráficos, tendo sido empregadas 20 repetições para cada propriedade avaliada.

Para a avaliação das características físico- mecânicas dos BLCs, foi empregado o delineamento inteiramente casualizado, com arranjo fatorial, em que foram analisados os efeitos dos fatores tratamento preservativo (Timbor e água); e adesivo empregado (emulsão de isocianato polimérico EPI; melamina-ureia-formaldeído - MUF; acetato de polivinila lincado - PVAc e resorcinol-formaldeído - RF). Em caso de significância pelo teste F ( $p \leq$ $0,05)$, foi procedido o teste Tukey $(\mathrm{p} \leq 0,05)$. Foram empregadas oito repetições para cada combinação de tratamento preservativo e adesivo. Para testar o percentual de falhas no bambu e a delaminação dos BLCs, foi necessário normalizar a distribuição dos dados pela transformação, sugerida por Steel e Torrie (1980).

\section{RESULTADOS E DISCUSSÃO}

\section{Densidade básica, retratibilidade volumétrica e curva de secagem dos bambus}

A densidade básica do bambu-gigante variou de 0,40 a $0,80 \mathrm{~g} . \mathrm{cm}^{-3}$, com média de 0,60 g. $\mathrm{cm}^{-3}$. Já o bambu-vulgar teve densidade básica entre 0,30 e 0,80 g. $\mathrm{cm}^{-3}$, com média de $0,50 \mathrm{~g} . \mathrm{cm}^{-}$ 3. Valores semelhantes de densidade para as duas espécies foram obtidos por Brito e Tomazello Filho (1987), Nascimento e Xavier (2012), Tiburtino (2012) e Brito (2013).

As duas espécies tiveram, praticamente, a mesma contração nas direções tangencial (largura) e radial (espessura), de aproximadamente $8 \%$ (Tabela 1). O comportamento observado para o bambu não ocorre com a madeira, que possui contração tangencial sempre maior que a radial. Contudo, a contração longitudinal teve o mesmo padrão encontrado para madeiras, podendo ser desconsiderada por causa do pequeno valor obtido. Entretanto, é importante ressaltar que o bambuvulgar possui maior contração volumétrica que o

TABELA 1: Valores médios de contração nas direções tangencial, radial, longitudinal e volumétrica dos bambus gigante e vulgar.

TABLE 1: Average values of contraction in radial, tangential, longitudinal and volumetric directions of Dendrocalamus giganteus and Bambusa vulgaris bamboos.

\begin{tabular}{ccccc}
\hline \multirow{2}{*}{ Espécie } & \multicolumn{4}{c}{ Contração (\%) } \\
\cline { 2 - 5 } & Tangencial & Radial & Longitudinal & Volumétrica \\
\hline Dendrocalamus & 7,81 & 7,64 & 0,27 & 15,72 \\
giganteus & 8,77 & 7,92 & 0,22 & 16,91 \\
Bambusa vulgaris & & \multicolumn{4}{c}{} \\
\hline
\end{tabular}


gigante.

A curva de secagem para as duas espécies de bambu é mostrada na Figura 2. As contrações fforam lineares até, 20 a $10 \%$ de umidade, quando ocorreu um ponto de inflexão na reta de secagem e passou-se a ter uma maior variação.

Desta maneira, pode-se identificar o ponto em que as fibras do bambu tiveram maior nível de contração, indicando a saída da água higroscópica da parede celular e consequentemente maior contração das fibras. Este ponto é conhecido para estudos com madeira, como o ponto de saturação das fibras (PSF) e, para os bambus gigante e vulgar ficou em torno de 10 a $20 \%$ de umidade.

Entretanto, também é importante frisar que a contração do bambu não seguiu as contrações comumente observadas em madeiras, nas quais quase não há alteração nas dimensões até a umidade atingir o PSF (ROSA, 2013). Isto não ocorre com o bambu, pois existiu uma variação linear e positiva desde o início da perda de umidade.

\section{Caracterização das propriedades físicas dos bambus laminados colados - BLCs}

\section{Densidade básica dos BLCs}

$\mathrm{Na}$ Tabela 2 constam os valores de densidade básica dos BLCs manufaturados com os bambus gigante e vulgar de acordo com cada tratamento. Os BLCs de bambu-gigante que foram tratados com água tiveram densidade média de 0,65 g. $\mathrm{cm}^{-3}$ e os tratados com Timbor a densidade foi de $0,60 \mathrm{~g} . \mathrm{cm}^{-3}$. Também se verifica que as densidades para os BLCs desta espécie, confeccionados com os diferentes adesivos, variaram de 0,59 a $0,65 \mathrm{~g} . \mathrm{cm}^{-3}$. Os valores de densidade dos BLCs produzidos foram semelhantes àqueles do bambu-gigante in natura. Assim, nota-se que os tratamentos preservativos e os adesivos não influenciaram na densidade básica dos BLCs. Isto ocorreu provavelmente em função da retenção de produto preservativo (Timbor) em $\mathrm{kg} . \mathrm{m}^{-3}$ de material ou da quantidade de amidos extraídos pela água, como também da massa de adesivos empregados na colagem das lâminas ser pequena em relação à massa dos bambus utilizados na pesquisa.

Os valores de densidade dos BLCs produzidos com bambu-gigante e tratados com água foram superiores aos obtidos por Rivero (2003), que variaram entre 0,51 a $0,54 \mathrm{~g} . \mathrm{cm}^{-3}$, mas inferiores aos encontrados Nogueira (2008), com média de 0,76 g. $\mathrm{cm}^{-3}$.

Para os BLCs confeccionados com bambuvulgar tratados com água, a densidade média foi

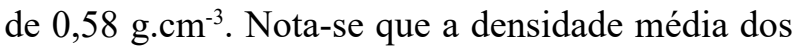
BLCs produzidos com os diferentes adesivos teve valores médios que variaram entre 0,54 a $0,61 \mathrm{~g} . \mathrm{cm}^{-}$ 3. Para esta espécie, houve influência dos adesivos na densidade dos BLCs. Os valores de densidade do bambu-vulgar in natura obtidos neste trabalho foram inferiores àqueles dos BLCs produzidos com esta mesma espécie.

\section{Inchamento dos BLCs}

Na Figura 3 é demonstrada a variação da espessura do BLC de Dendrocalamus giganteus

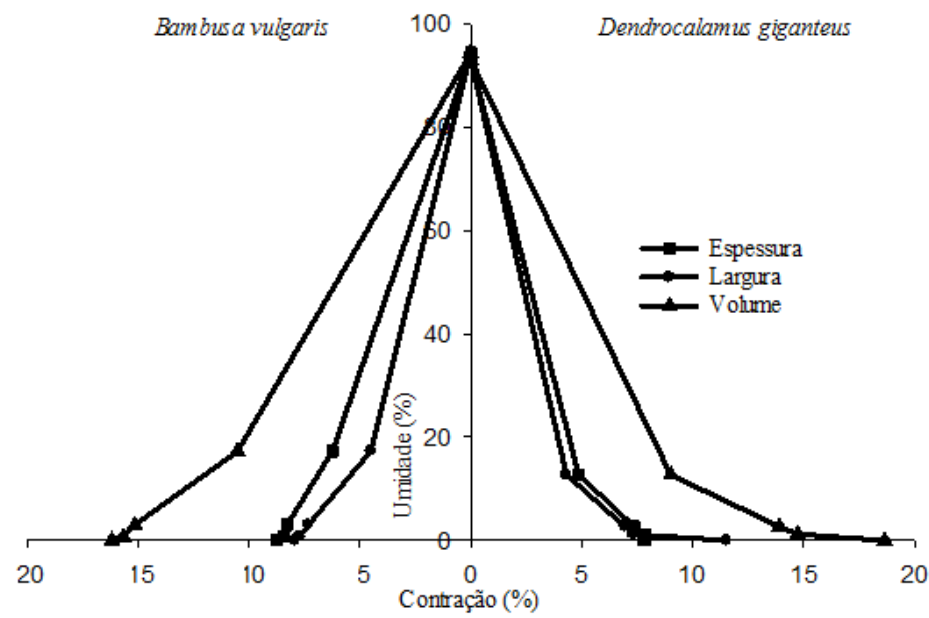

FIGURA 2: Curvas de secagem e contração para os bambus gigante e vulgar.

FIGURE 2: Drying curves and shrinkage for Dendrocalamus giganteus and Bambusa vulgaris bamboos. 
TABELA 2: Densidade básica dos BLCs produzidos com bambus gigante e vulgar tratados com água ou Timbor para os diferentes adesivos testados.

TABLE 2: Basic density of LBLs manufactured with Dendrocalamus giganteus and Bambusa vulgaris treated with water or Timbor for different tested adhesives.

\begin{tabular}{|c|c|c|c|c|c|c|}
\hline Espécie & & Adesiy & ensidade & ásica (g & $\left.n^{-3}\right)$ & \\
\hline \multirow{4}{*}{ Dendrocalamus giganteus } & Tratamento & EPI & MUF & PVA & $\mathrm{RF}$ & Média \\
\hline & Água & $\begin{array}{c}0,70 \\
(0,03)\end{array}$ & $\begin{array}{c}0,60 \\
(0,04)\end{array}$ & $\begin{array}{c}0,63 \\
(0,10)\end{array}$ & $\begin{array}{c}0,65 \\
(0,03)\end{array}$ & $\begin{array}{c}0,65 \\
(0,07)\end{array}$ \\
\hline & Timbor & $\begin{array}{c}0,60 \\
(0,04)\end{array}$ & $\begin{array}{c}0,57 \\
(0,05)\end{array}$ & $\begin{array}{c}0,58 \\
(0,04)\end{array}$ & $\begin{array}{c}0,64 \\
(0,08)\end{array}$ & $\begin{array}{c}0,60 \\
(0,06)\end{array}$ \\
\hline & Média & $\begin{array}{c}0,65 \\
(0,06)\end{array}$ & $\begin{array}{c}0,59 \\
(0,05)\end{array}$ & $\begin{array}{c}0,61 \\
(0,08)\end{array}$ & $\begin{array}{c}0,65 \\
(0,06)\end{array}$ & \\
\hline \multirow{4}{*}{ Bambusa vulgaris } & Tratamento & EPI & MUF & PVA & RF & Média \\
\hline & Água & $\begin{array}{c}0,56 \\
(0,02) \\
\end{array}$ & $\begin{array}{c}0,61 \\
(0,03) \\
\end{array}$ & $\begin{array}{c}0,52 \\
(0,04) \\
\end{array}$ & $\begin{array}{c}0,62 \\
(0,06) \\
\end{array}$ & $\begin{array}{c}0,58 \\
(0,04) \\
\end{array}$ \\
\hline & Timbor & $\begin{array}{c}0,56 \\
(0,02)\end{array}$ & $\begin{array}{c}0,61 \\
(0,5)\end{array}$ & $\begin{array}{c}0,56 \\
(0,05)\end{array}$ & $\begin{array}{c}0,61 \\
(0,02)\end{array}$ & $\begin{array}{c}0,58 \\
(0,04)\end{array}$ \\
\hline & Média & $\begin{array}{c}0,56 \\
(0,02)\end{array}$ & $\begin{array}{c}0,61 \\
(0,04)\end{array}$ & $\begin{array}{c}0,54 \\
(0,05)\end{array}$ & $\begin{array}{c}0,61 \\
(0,04)\end{array}$ & \\
\hline
\end{tabular}

Em que: $\mathrm{EPI}=$ emulsão de isocianato polimérico; $\mathrm{MUF}=$ melamina-ureia-formaldeído; $\mathrm{PVAc}=$ acetato de polivinila lincado e RF = resorcinol-formaldeído. Valores entre parênteses é o desvio padrão.

e de Bambusa vulgaris em função do tempo de saturação, dos tratamentos preservativos e dos adesivos utilizados. No lado esquerdo da figura consta a variação da espessura dos BLCs tratados com água com os respectivos adesivos e, no lado direito, a variação da espessura dos BLCs tratados com Timbor.

Pode-se notar que todos os BLCs, tanto aqueles tratados com água ou com Timbor para as duas espécies, tiveram o mesmo padrão de inchamento, aumentando sua espessura até 48 horas de saturação, com tendência à estabilização depois deste tempo. Entretanto, pode ser observado que essa estabilização não é evidente no bambu-vulgar.

Os BLCs produzidos com o bambu-gigante, tratados com água e colados com o PVA, tiveram o maior inchamento $(6,13 \%)$. Para aqueles aderidos com EPI, o inchamento foi de 5,42\%, com MUF de $5,64 \%$ e com RF de $4,50 \%$, que proporcionou a maior estabilidade dimensional para os BLCs. Já para os BLCs da mesma espécie e tratados com Timbor, destacam-se os aderidos com PVA, que tiveram o inchamento superior aos demais, de $6,96 \%$. Já os aderidos com RF possuíram o menor inchamento $(3,97 \%)$. Em resumo, vale ressaltar que os BLCs produzidos com o bambu-gigante e colados com PVA e RF, tratados com água ou com
Timbor, possuíram o maior e o menor inchamento, respectivamente. De forma similar, os BLCs produzidos com bambu-gigante, colados com PVA e tratados com água ou com Timbor, tiveram os maiores índices de inchamento na espessura.

Ainda na Figura 3, observa-se que os BLCs produzidos com o bambu-vulgar, tratados com água e colados com EPI e PVA, tiveram o mesmo comportamento e a maior variação na espessura, de 10,29 e $9,65 \%$ respectivamente. Porém, para aqueles colados com MUF e RF, o inchamento foi de 6,94 e $5,59 \%$, com este último adesivo proporcionando um comportamento mais estável aos BLCs. Assim como ocorreu um aumento significativo na espessura para os BLCs de bambu-gigante tratados com Timbor e aderidos com PVA, também ocorreu para os mesmos BLCs de bambu-vulgar, ou seja, preservados com Timbor e colados com PVA. Este inchamento foi de $11,9 \%$ devido à baixa resistência à umidade do adesivo PVA. Os BLCs aderidos com EPI, MUF e RF tiveram o mesmo comportamento e o inchamento final foi de 6,68,6,28 e 6,48\%, respectivamente. Em síntese, os BLCs produzidos com o bambu-vulgar, colados com PVA e tratados com água ou com Timbor, possuíram os maiores índices de inchamento na espessura.

Na Figura 4 é apresentada a variação da 

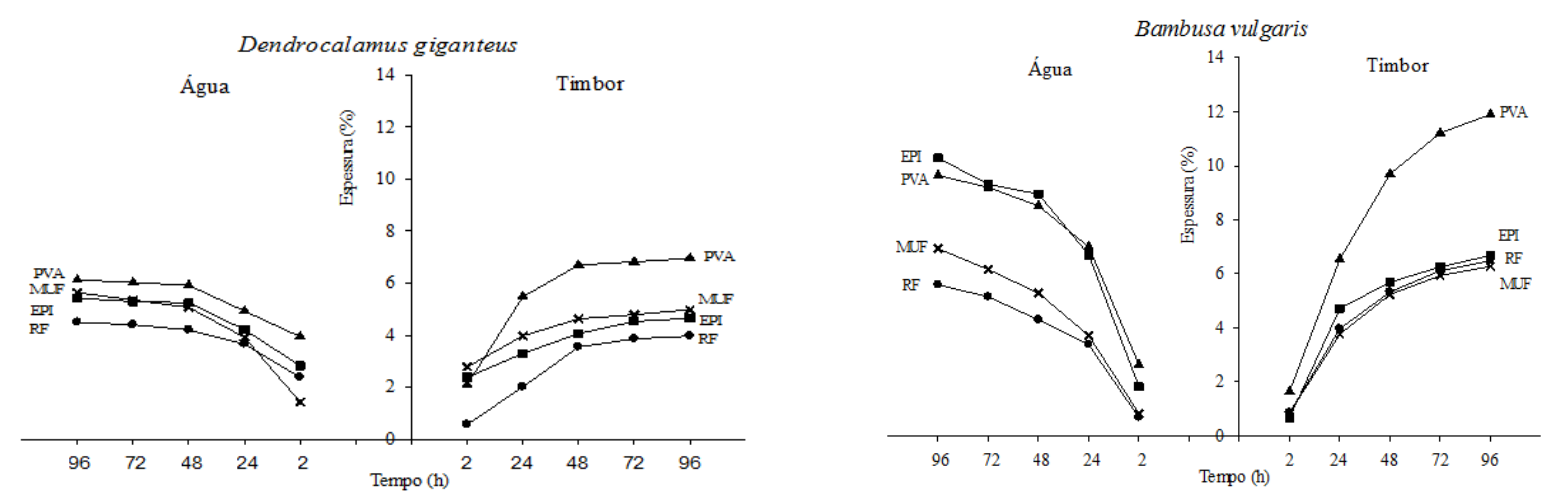

Em que: $\mathrm{EPI}=$ emulsão de isocianato polimérico; $\mathrm{MUF}=$ melamina-ureia-formaldeído; $\mathrm{PVAc}=$ acetato de polivinila lincado e RF = resorcinol-formaldeído.

FIGURA 3: Inchamento médio da espessura dos BLCs produzidos com os bambus gigante e vulgar conforme o tempo de imersão em água.

FIGURE 3: Average thickness swelling of LBLs produced with Dendrocalamus giganteus and Bambusa vulgaris bamboos according to the time of water immersion.
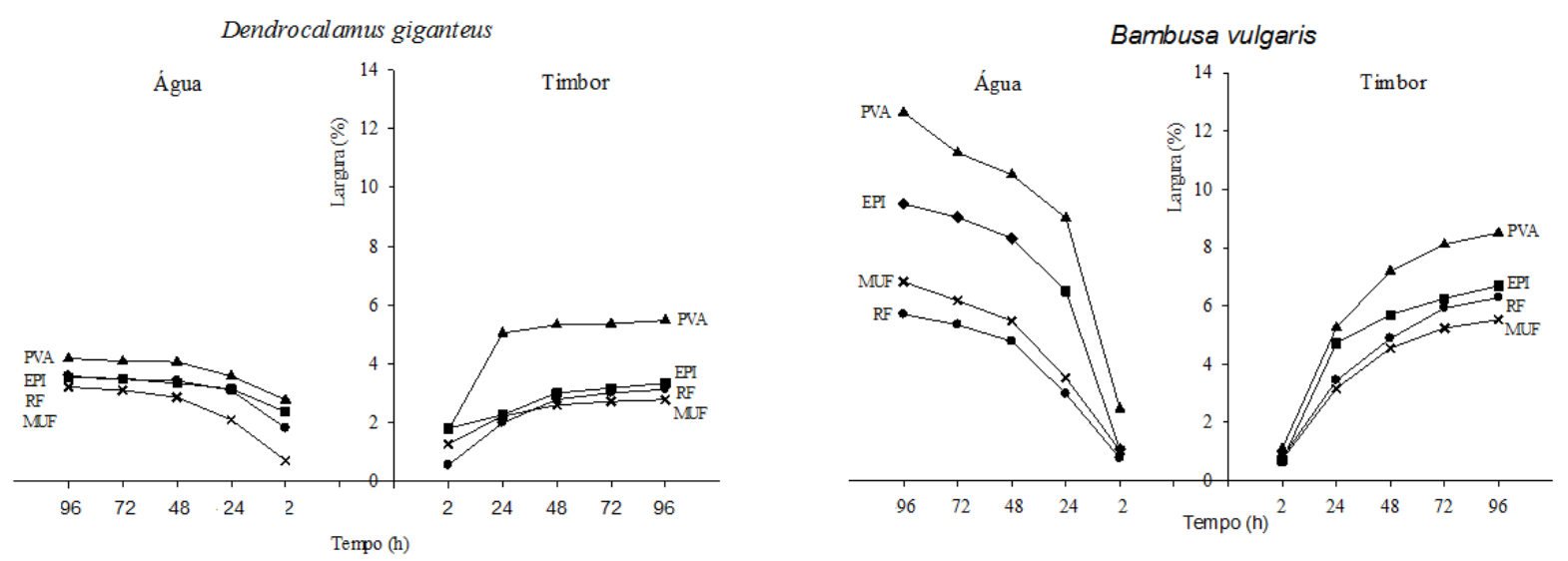

Em que: $\mathrm{EPI}=$ emulsão de isocianato polimérico; $\mathrm{MUF}=$ melamina-ureia-formaldeído; $\mathrm{PVAc}=$ acetato de polivinila lincado e RF = resorcinol-formaldeído.

FIGURA 4: Inchamento médio da largura dos BLCs produzidos com os bambus gigante e vulgar, conforme o tempo de imersão em água.

FIGURE 4: Average width swelling of LBLs produced with Dendrocalamus giganteus and Bambusa vulgaris bamboos according to the water immersion time.

largura do BLC dos bambus gigante e vulgar, conforme o tempo de imersão em água, dos tratamentos preservativos e dos adesivos utilizados. Assim como ocorreu com o inchamento da espessura dos BLCs de bambu-gigante e de bambuvulgar tratados com água ou com Timbor, o mesmo padrão de inchamento foi verificado para a largura. Essa dimensão aumentou até 48 horas de saturação, com tendência à estabilização após este tempo de imersão. Contudo, pode ser observado que essa estabilização também é menos consolidada no bambu-vulgar, caracterizando menor estabilidade dimensional para esta espécie.

Os BLCs produzidos com bambu-gigante tratados com água e aderidos com MUF tiveram a menor variação na largura, de 3,21\%. Aqueles BLCs colados com EPI e RF demonstram o mesmo comportamento no inchamento, chegando a 3,54 e $3,58 \%$, respectivamente, enquanto os BLCs colados com PVA demonstraram o maior inchamento, de $4,18 \%$. Assim como também ocorreu no inchamento em espessura, os BLCs do bambu-gigante tratado com Timbor e aderidos com PVA demonstraram o inchamento expressivamente maior que os outros, 
de 5,47\%. Aqueles BLCs colados com MUF tiveram um comportamento mais estável $(2,78 \%)$ e os aderidos com EPI e RF o mesmo comportamento na variação da largura, chegando a 3,34 e 3,13\% respectivamente. Em resumo, os BLCs produzidos com o bambu-gigante e colados com PVA e MUF, tratados tanto com água quanto com Timbor, tiveram o maior e o menor inchamento, respectivamente.

Ainda na Figura 4 nota-se que os BLCs produzidos com o bambu-vulgar, tratados comágua e colados com EPI e PVA, tiveram um comportamento similar com a maior variação na largura, de 9,50 e $12,64 \%$, respectivamente. Os BLCs aderidos com MUF obtiveram inchamento de $6,83 \%$ e os colados com RF tiveram maior estabilidade, com 5,70\% de inchamento. Assim como ocorreu um aumento significativo na largura para os BLCs de bambugigante tratados com Timbor e aderidos com PVA, também ocorreu para os BLCs de bambu-vulgar tratados com Timbor e colados com PVA, com $8,51 \%$ de inchamento. Para aqueles BLCs colados com EPI e RF, o inchamento foi de 6,09 e 6,27\%. Já os BLCs aderidos com MUF tiveram o menor inchamento, de 5,52\%. Assim, em ambas as espécies tratadas com água ou com Timbor, o adesivo PVA foi o que proporcionou a menor estabilidade para a largura. Resultado semelhante foi obtido por Paes et al. (2009) ao compararem a estabilidade dimensional do BLC produzido com bambu-gigante e aderido com RF e PVA. Para os demais adesivos testados, não se dispõe de informações na literatura.

Na Figura 5 é apresentada a variação do volume dos BLCs produzidos com bambus gigante $\mathrm{e}$ vulgar em função do tempo de imersão em água, dos tratamentos preservativos e dos adesivos utilizados. Como o inchamento da espessura e da largura dos BLCs das duas espécies, tratadas com água ou com Timbor demonstraram o mesmo comportamento, logicamente era esperado que o volume também seguisse esta tendência, aumentando linearmente até 48 horas de imersão em água e tendendo a estabilizar após esse período. Além disto, também pode ser observado na Figura 4 que o bambuvulgar possui menor estabilidade dimensional que o bambu-gigante.

Os BLCs produzidos com bambugigante, tratados com água e aderidos com PVA possuíram a maior variação volumétrica, de $10,56 \%$. Aqueles colados com EPI e MUF tiveram o mesmo comportamento, com inchamentos de 9,12 e $9,01 \%$, respectivamente. Aqueles aderidos com RF demonstraram a maior estabilidade, com inchamento de $8,27 \%$. Para os BLCs produzidos com essa mesma espécie e tratados com Timbor, seguiu o mesmo padrão daqueles tratados com água, ou seja, os BLCs colados com PVA tiveram maior inchamento $(12,80 \%)$, aqueles colados com EPI e MUF demonstraram o mesmo comportamento no inchamento $(8,12$ e $7,95 \%)$ e os colados com RF exibiram o menor inchamento $(7,27 \%)$.

Ainda na Figura 5, verificou-se que os BLCs produzidos com o bambu-vulgar, tratados com água e colados com EPI ou PVA, tiveram um comportamento semelhante com a maior variação no volume, de 20,20 e $22,92 \%$. Os BLCs aderidos com MUF demonstraram inchamento de $14,19 \%$ e, os colados com RF maior estabilidade, com $11,66 \%$ de inchamento. Assim como ocorreu um aumento significativo no volume para os BLCs de bambugigante tratados com Timbor e aderidos com PVA, também ocorreu para os BLCs de bambu-vulgar, com $20,75 \%$ de inchamento. Para aqueles BLCs colados com EPI ou RF, o inchamento foi semelhante, de 13,07 e 13,01\%, respectivamente, enquanto os BLCs aderidos com MUF tiveram o menor inchamento, de $12,10 \%$. Em resumo, para ambas as espécies tratadas com água ou com Timbor, o adesivo PVA proporcionou a menor estabilidade volumétrica, enquanto os adesivos MUF e RF proporcionaram a maior estabilidade.

Brito (2013) obteve valor de $16,82 \%$ para o inchamento volumétrico máximo para o BLC confeccionado com o bambu-gigante e aderido com RF. Paes et al. (2009) obtiveram valores de inchamento máximo de 21,51 e de $20,00 \%$ para os BLCs confeccionados com bambu da mesma espécie, para os adesivos PVAe RF, respectivamente.

Com base nos resultados obtidos os BLCs produzidos com os bambus gigante e vulgar e aderidos com os adesivos MUF e RF poderiam ser empregados em situações que exigem maior estabilidade dimensional, como em móveis e pisos ou em locais que estariam sujeitos à maior variação de umidade.

\section{Ensaio de delaminação dos BLCs}

$\mathrm{Na}$ Tabela 3 pode ser observada a porcentagem de delaminação para cada BLC em função dos tratamentos e dos adesivos. Para os BLCs produzidos com bambu-gigante, a interação entre os fatores tratamentos e adesivos não foi significativa pelo teste $\mathrm{F}$ a $95 \%$ de probabilidade, o que permitiu a interpretação dos dados estatísticos 

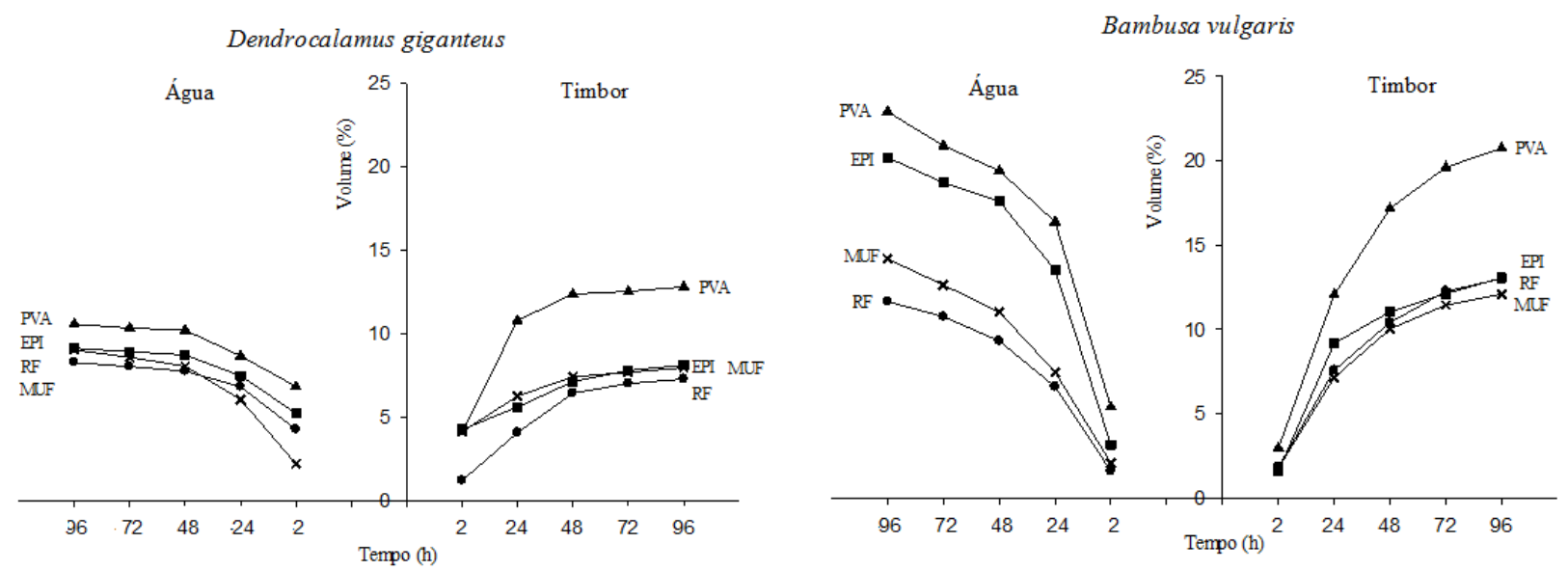

Em que: EPI = emulsão de isocianato polimérico; MUF = melamina-ureia-formaldeído; PVAc = acetato de polivinila lincado e RF = resorcinol-formaldeído.

FIGURA 5: Inchamento médio do volume dos BLCs produzidos com os bambus gigante e vulgar em função do tempo de imersão em água.

FIGURE 5: Average volume swelling of LBLs produced with Dendrocalamus giganteus and Bambusa vulgaris bamboos according to the water immersion time.

separadamente para cada fator. Deste modo, os BLCs desta espécie tratados com água ou com Timbor tiveram, estatisticamente, a mesma delaminação, de 18,86 e $19,58 \%$, respectivamente.

Os BLCs de bambu-gigante que tiveram o maior índice de delaminação foram os unidos com EPI, de $41,35 \%$ seguidos daqueles aderidos com PVA, de 30,09\%. Aqueles colados com MUF demonstraram apenas $5,42 \%$ e os aderidos com RF não tiveram delaminação, o que comprova a alta resistência à umidade destas resinas.

Os BLCs de bambu-vulgar demonstraram interação entre os fatores tratamento e adesivos (Tabela 5). Assim, os BLCs colados com EPI e tratados com água tiveram maior delaminação do que aqueles tratados com Timbor, 30,18 e 11, 44\%, respectivamente. Para aqueles BLCs unidos com PVA os resultados foram o inverso, ou seja, aqueles tratados com água tiveram menor porcentagem de delaminação que os tratados com Timbor, de 28,90 e 62,20\%. Já os BLCs unidos com MUF e RF para ambos os tratamentos, não tiveram delaminação.

Os BLCs confeccionados com bambuvulgar, em particular aqueles que foram tratados com água, os adesivos EPI, MUF e RF tiveram, estatisticamente, o mesmo comportamento de delaminação, de 11,$44 ; 0,00$ e $0,00 \%$, respectivamente, enquanto aqueles colados com PVA tiveram 62,20\% de delaminação (Tabela 3), indicando que este adesivo não é apropriado para aderir juntas a serem empregadas em uso externo.

\section{CONCLUSÕES}

As espécies de bambus gigante e vulgar tiveram, praticamente, a mesma contração nas direções tangenciais e radiais. O ponto de saturação das fibras para as duas espécies ficou em torno de 10 a $20 \%$ de umidade.

As densidades dos BLCs confeccionados com a espécie bambu-gigante não foram afetadas pelos tratamentos preservativos ou adesivos utilizados. O mesmo não ocorreu para aqueles BLCs produzidos com bambu-vulgar, em que os valores de densidade foram influenciados pelo tipo de adesivo utilizado.

Os BLCsconfeccionados combambu-vulgar demonstraram menor estabilidade dimensional que os produzidos com o bambu-gigante.

Os adesivos melamina-ureia-formaldeído (MUF) e resorcinol-formaldeído (RF) conferiram maior estabilidade dimensional aos BLCs produzidos com ambas as espécies.

Tanto os BLCs de bambu-gigante quanto os de vulgar com relação aos dois tratamentos preservativos, tiveram delaminação superior àqueles aderidos com a emulsão de isocianato polimérico (EPI) e acetato de polivinila (PVA), demonstrando a ineficiência dos adesivos na colagem dos bambus para uso externo.

Apenas os BLCs aderidos com melaminaureia-formaldeído (MUF) e resorcinol-formaldeído (RF), de ambas as espécies, não ultrapassaram a 
TABELA 3: Delaminação dos BLCs produzidos com os bambus gigante e vulgar tratados com água ou Timbor para os diferentes adesivos.

TABLE 3: LBLs delamination manufactured with Dendrocalamus giganteus and Bambusa vulgaris bamboos treated with water or Timbor for different adhesives.

\begin{tabular}{ccccccc}
\hline \multirow{2}{*}{ Espécie } & \multicolumn{7}{c}{ Adesivos/Delaminação (\%) } \\
\cline { 2 - 7 } & Tratamento & EPI & MUF & PVA & RF & Média \\
\hline \multirow{3}{*}{ Dendrocalamus giganteus } & Água & 45,09 & 4,05 & 26,29 & 0,00 & $18,86 \mathrm{a}$ \\
& Timbor & 37,61 & 6,80 & 33,90 & 0,00 & $19,58 \mathrm{a}$ \\
& Média & $41,35 \mathrm{~A}$ & $5,42 \mathrm{C}$ & $30,09 \mathrm{~B}$ & $0,00 \mathrm{C}$ & \\
\hline \multirow{3}{*}{ Bambusa vulgaris } & Tratamento & EPI & MUF & PVA & RF & Média \\
\hline & Água & $30,18 \mathrm{Aa}$ & $0,00 \mathrm{Ba}$ & $28,90 \mathrm{Ab}$ & $0,00 \mathrm{Ba}$ & 14,77 \\
& Timbor & $11,44 \mathrm{Bb}$ & $0,00 \mathrm{Ba}$ & $62,20 \mathrm{Aa}$ & $0,00 \mathrm{Ba}$ & 18,41 \\
& Média & 20,81 & 0,00 & 45,55 & 0,00 & \\
\hline
\end{tabular}

Em que: $\mathrm{EPI}=$ emulsão de isocianato polimérico; $\mathrm{MUF}=$ melamina-ureia-formaldeído; $\mathrm{PVAc}=$ acetato de polivinila lincado; $\mathrm{RF}$ = resorcinol-formaldeído. Médias seguidas da mesma letra minúscula na linha ou minúsculas na coluna, não diferiram em entre si pelo teste Tukey $(\mathrm{p}>0,05)$.

delaminação exigida na norma, estando aptos para suportar as condições de intemperismo que ocorrem ambientes externos.

\section{AGRADECIMENTOS}

À Fundação de Apoio à Ciência e Tecnologia do Espírito Santo (FAPES) pelo apoio financeiro, Processo 49781880, Edital FAPES 001/201- Tecnologias Sociais, pela concessão de bolsa de estudo ao primeiro autor e à Empresa AkzoNobel pela doação dos adesivos empregados nesta pesquisa. Aos senhores Sebastião de Azevedo dos Santos e Ronaldo Silveira Ribeiro, pela doação dos bambus.

\section{REFERÊNCIAS BIBLIOGRÁFICAS}

AMERICAN INSTITUTE OF TIMBER CONSTRUCTION. AITC test T110: cyclic delamination test. Centennial, p. 19 - 21, 2007.

AMERICAN SOCIETY FOR TESTING AND MATERIALS. ASTM D-143: standard test methods for small clear specimens of timber. Philadelphia, 2005. 31p.

ASSOCIAÇÃO BRASILEIRA DE NORMAS TÉCNICAS - ABNT. NBR 7190: projeto de estruturas de madeira. Rio de Janeiro, 1997. 107p.

AZZINI, A.; BERALDO. A.L. Métodos práticos para utilização do bambu. Campinas: Universidade de Campinas, 2001. 14p.

BRITO, F.M.S. Efeito da termorretificação nas propriedades tecnológicas do bambu. 2013. 88f. Dissertação (Mestrado em Ciências Florestais) Universidade Federal do Espírito Santo, Jerônimo Monteiro, 2013.

BRITO, J. O.; TOMAZELLO FILHO, M. Produção e caracterização do carvão vegetal de espécies e variedades de bambu. IPEF, Piracicaba, n.36, p.13-17, 1987.

COLLA, W. A. Efeito do tratamento térmico nas características físicas e mecânicas do bambu Dendrocalamus giganteus (Munro). 2010. $111 \mathrm{f}$. Dissertação (Mestrado em Construções Rurais e Ambiência) - Universidade Estadual de Campinas, Campinas, 2010.

GARCIA, N. País dá primeiros passos no uso industrial do bambu. Revista da Rede Brasileira do Bambu, Brasília, n.1, p.14-15, 2010.

LEPAGE, E. S. Preservativos e sistemas preservativos. In: LEPAGE, E. S. (Coord.). Manual de preservação de madeiras. São Paulo: IPT, 1986. v.1, p.279-314.

MOIZÉS, F.A. Painéis de bambu, uso e aplicações: uma experiência didática nos cursos de Design em Bauru, São Paulo. 2007. 116f. Dissertação (Mestrado em Desenho Industrial) - Universidade Estadual Paulista, Bauru, 2007.

NASCIMENTO, A.M.; XAVIER, L.M. Densidade básica e retratibilidade volumétrica de 
Dendrocalamus giganteus, Bambusa tuldoides, Bambusa vulgaris e Phyllostachys aurea. In: ENCONTRO BRASILEIRO EM MADEIRAS E EM ESTRUTURAS DE MADEIRA, 13., Vitória, 2012. Anais... Vitória: UFES, 2012. CD ROM.

NOGUEIRA, C. L. Painel de bambu laminado colado estrutural. 2008. 94f. Dissertação (Mestrado em Recursos Florestais) - Universidade de São Paulo, Escola Superior de Agricultura "Luiz de Queiroz". Piracicaba, 2008.

MORÁN UBIDIA, J.A. Preservación del bambú en América Latina, mediante métodos tradicionales. Guayaquil: International Network for Bamboo and Rattan - INBAR, 2002.70p. (Technical Report, 25). Disponível em: < http:// www.inbar.int/publication/ txt/ INBAR_Technical_Report_No25.htm $>$. Acesso em: 19 set. 2014.

OBATAYA, E.; KITIN, P.; YAMAUCHI, H. Bending characteristics of bamboo (Phyllostachys pubescens) with respect to its fiber-foam composite structure. Wood Science and Technology, New York, n.41, p.385-400, 2007.

OLIVEIRA, A.K.F. Caracterização físicomecânica de laminado colado de bambu (Dendrocalamus giganteus) para revestimento de pisos. 2005. 75f. Dissertação (Mestrado em Engenharia Agrícola) - Universidade Federal de Campina Grande, Campina Grande, 2005.

PAES, J.B.; et al. Caracterização físico-mecânica do laminado colado de bambu (Dendrocalamus giganteus). Ciência Florestal, Santa Maria, v. 19, n. 1, p. 41-51, 2009.

PAES, J.B. et al. Caracterização e aspecto de um piso confeccionado com bambu (Dendrocalamus giganteus Munro). Ciência da Madeira, Pelotas, v. 1, n. 1, p. 52-67, 2010.

PEREIRA, M.A.R; BERALDO, A.L. Bambu de corpo e alma. Bauru: Canal 6, 2008. 240p.

QISHENG, Z.; SHENXUE, J; YONGYU, T. Industrial utilization on bamboo. Beijing: International Network for Bamboo and Rattan, 2002. (Nota Técnica).

RIVERO, L.A. Laminado colado e contraplacado de bambu. 2003. 83f. Dissertação (Mestrado em Engenharia Agrícola) - Universidade de Campinas, Campinas, 2003.

ROSA, R.A. Caracterização do bambu laminado colado como alternativa tecnológica industrial. 2013. 80f. Dissertação (Mestrado em Ciências Florestais) - Universidade Federal do Espírito Santo, Jerônimo Monteiro, 2013.

SANTOS, R.L.; LOPES, J.D.S. Construções com bambu: opção de baixo custo. Viçosa: Centro de Produções Técnica, 1998. 40 p. (Série Construções Rurais, 160).

STEEL, R.G.D.; TORRIE, J.H. Principles and procedures of statistic: a biometrical approach. 2 . ed. New York: Mc-Graw Hill, 1980. 633 p.

TIBURTINO, R.F. Qualidade e eficiência do tratamento preservativo de duas espécies de bambu. 2012. 106f. Dissertação (Mestrado em Ciências Florestais) - Universidade Federal do Espírito Santo, Jerônimo Monteiro, 2012.

VAZ, D. China: a civilização do bambu. Revista da Rede Brasileira do Bambu, Brasília, n.1, p.26-27, 2010. 